\title{
EXPRESSION OF ERYTHROPOIETIN AND ERYTHROPOIETIN RECEPTOR ON RENAL TISSUE IN CHILDREN WITH ACUTE KIDNEY INJURY
}

\author{
Z. Li, T. Hui, M. Xun, C. Duan, Y. Zhang, Y. Yin, Y. Ding, L. Zhang \\ Nephrology, Hunan Children's Hospital, Changsha, China
}

Objective: To detect relationship between expression of erythropoietin (EPO) and erythropoietin receptor (EPOR) on kidney and kidney lesion in children with acute kidney injury (AKI).

Methods: Thirty-eights children with AKI who involved in the study. The patient population comprised 29 boys and 9 girls. The children with thin basement membrane nephropathy were as control in the study. The expression of EPO and EPOR on kidney in AKI children were examined by immunity histochemistry method. The renal tubulointerstitium histopathologic lesion was shown by the counting score. The correlations between EPO and EPOR expression levels and histopathologic lesion degrees in renal tubulointerstitium were studied in children with AKI. Results Thirty-eight children with AKI were divided into mild patients ( AKI 1 and AKI 2) and serious patients (AKI 3). The levels of EPO expression in AKI children kidney were $(6.52 \pm 2.12) \%,(3.02 \pm 0.79) \%$, and $(1.62 \pm 0.18) \%(\mathrm{P}<0.01)$, respectively, in control group, mild patients, serious patients. The levels of EPOR expression in AKI children kidney were $40.46 \pm 8.42) \%, 64.78 \pm 16.38) \%, 62.36 \pm 15.67) \%(\mathrm{P}<0.01)$, respectively, in control group, mild patients, serious patients. There were negative correlation between EPO expression and histopathologic lesion degrees in kidney $(\mathrm{r}=-0.872, \mathrm{P}<0.01)$. There were positive correlation between EPOR expression and histopathologic lesion degrees in kidney $(\mathrm{r}=0.772, \mathrm{P}<0.01)$.

Conclusion: The level of EPO secretion was decreased, but the expression of EPOR was increased in AKI children kidney, which suggested that using extraneous EPO may help to renal repair of AKI children. 